Krzysztof Burnecki (Wrocław)

PaWę Miśta (Wrocław)

Aleksander Weron (Wrocław)

\title{
WHAT IS THE BEST APPROXIMATION OF RUIN PROBABILITY IN INFINITE TIME?
}

Abstract. We compare 12 different approximations of ruin probability in infinite time studying typical light- and heavy-tailed claim size distributions, namely exponential, mixture of exponentials, gamma, lognormal, Weibull, loggamma, Pareto and Burr. We show that approximation based on the Pollaczek-Khinchin formula gives most accurate results, in fact it can be chosen as a reference method. We also introduce a promising modification to the De Vylder approximation.

1. Introduction. In a very interesting paper Grandell (2000) demonstrates that between possible simple approximations of ruin probabilities in infinite time the most successful is the De Vylder approximation, which is based on the idea to replace the risk process with the one with exponentially distributed claims and ensuring that the first three moments coincide.

In Section 2 we recall exact results on the ruin probability in infinite time. Next, in Section 3 we present 12 different approximations of ruin probability in infinite time. We introduce a modification to the De Vylder approximation by changing the exponential to the gamma distribution and fitting the first three moments. This modification is promising and works in many cases better than the original method. Second, in contrast to Grandell's paper, we drop the "simplicity" assumption and show in Section 4 that approximation based on the Pollaczek-Khinchin formula gives the best results. Moreover, it works for all possible distributions of claims and can be chosen as a reference method (see Section 5).

2000 Mathematics Subject Classification: 62P05, 60G55.

Key words and phrases: risk process, ruin probability, loss distribution, PollaczekKhinchin formula, De Vylder approximation. 
We start with the definition of a classical risk model (see e.g. Grandell, 1991, and Rolski et al., 1999). Let $(\Omega, \mathcal{F}, \mathbb{P})$ be a probability space carrying a Poisson process $\left\{N_{t}\right\}_{t \geq 0}$ with intensity $\lambda$, and a sequence $\left\{X_{k}\right\}_{k=1}^{\infty}$ of independent, positive, identically distributed random variables, with mean $\mu$ and variance $\sigma^{2}$. Furthermore, we assume that $\left\{X_{k}\right\}$ and $\left\{N_{t}\right\}$ are independent. The classical risk process $\left\{R_{t}\right\}_{t \geq 0}$ is given by

$$
R_{t}=u+c t-\sum_{i=1}^{N_{t}} X_{i},
$$

where $c$ is some positive constant and $u$ is nonnegative.

This is the standard mathematical model for insurance risk. The initial capital is $u$, the Poisson process $N_{t}$ describes the number of claims in the $(0, t]$ time interval, and claim severities are random, given by the sequence $\left\{X_{k}\right\}_{k=1}^{\infty}$. To cover its liability, the insurance company receives a premium at a constant rate $c$, per unit time, where $c=(1+\theta) \lambda \mu$ and $\theta>0$ is often called the relative safety loading. The loading has to be positive, otherwise $c$ would be less than $\lambda \mu$ and thus with probability 1 the business risk would become negative in infinite time.

For mathematical purposes, it is sometimes more convenient to work with a claim surplus process $\left\{S_{t}\right\}_{t \geq 0}$ (see e.g. Asmussen, 2000), namely

$$
S_{t}=u-R_{t}=\sum_{i=1}^{N_{t}} X_{i}-c t .
$$

Now, we recall the definition of ruin probability, i.e. the probability that the risk process drops below zero. The time to ruin is defined as

$$
\tau(u)=\inf \left\{t \geq 0: R_{t}<0\right\}=\inf \left\{t \geq 0: S_{t}>u\right\} .
$$

Let

$$
M=\sup _{0 \leq t<\infty}\left\{S_{t}\right\}, \quad M_{T}=\sup _{0 \leq t \leq T}\left\{S_{t}\right\} .
$$

DeFINITION 1.1. The ruin probability in finite time is given by

$$
\psi(u, T)=\mathbb{P}(\tau(u) \leq T)=\mathbb{P}\left(M_{T}>u\right)
$$

and the ruin probability in infinite time is defined as

$$
\psi(u)=\mathbb{P}(\tau(u)<\infty)=\mathbb{P}(M>u) .
$$

In what follows we assume $c=1$, but this is not a restrictive assumption. Following Asmussen (2000), let $c \neq 1$ and define $\widetilde{R}_{t}=R_{t / c}$. Then the relations between the ruin probabilities $\psi(u), \psi(u, T)$ for the process $R_{t}$ and $\widetilde{\psi}(u), \widetilde{\psi}(u, T)$ for the process $\widetilde{R}_{t}$ are given by the equations

$$
\psi(u)=\widetilde{\psi}(u), \quad \psi(u, T)=\widetilde{\psi}(u, T c) .
$$


In actuarial mathematics we distinguish between light- and heavy-tailed distributions (see e.g. Embrechts et al., 1997). A distribution $F_{X}(x)$ is said to be light-tailed if there exist constants $a, b>0$ such that $\bar{F}_{X}(x)=1-F_{X}(x) \leq$ $a e^{-b x}$ or, equivalently, if there exists $z>0$ such that $M_{X}(z)<\infty$, where $M_{X}(z)$ is the moment generating function. A distribution $F_{X}(x)$ is said to be heavy-tailed if $\bar{F}_{X}(x)>a e^{-b x}$ for all $a, b>0$, or, equivalently, if $M_{X}(z)=\infty$ for all $z>0$.

In this paper we study claim size distributions as in Table 1.

Table 1. Claim size distributions

\begin{tabular}{llll}
\hline \multicolumn{3}{c}{ Light-tailed distributions } \\
\hline Name & Parameters & Pdf & \\
\hline exponential & $\beta>0$ & $f_{X}(x)=\beta e^{-\beta x}$, & $x \geq 0$ \\
gamma & $\alpha>0, \beta>0$ & $f_{X}(x)=\frac{\beta^{\alpha}}{\Gamma(\alpha)} x^{\alpha-1} e^{-\beta x}$, & $x \geq 0$ \\
Weibull & $c>0, \tau \geq 1$ & $f_{X}(x)=c \tau x^{\tau-1} e^{-c x^{\tau}}$, & $x \geq 0$ \\
mixed exp's & $\beta_{i}>0, \sum_{i=1}^{n} a_{i}=1$ & $f_{X}(x)=\sum_{i=1}^{n}\left(a_{i} \beta_{i} e^{-\beta_{i} x}\right)$, & $x \geq 0$ \\
\hline \multicolumn{4}{c}{ Heavy-tailed distributions } \\
\hline Name & Parameters & Pdf & \\
\hline Weibull & $c>0,0<\tau<1$ & $f_{X}(x)=c \tau x^{\tau-1} e^{-c x^{\tau}}$, & $x \geq 0$ \\
lognormal & $\mu \in \mathbb{R} \sigma>0$ & $f_{X}(x)=\frac{1}{\sqrt{2 \pi} \sigma x} e^{-(\log (x)-\mu)^{2} / 2 \sigma^{2}}$, & $x \geq 0$ \\
loggamma & $\alpha>0, \beta>0$ & $f_{X}(x)=\frac{\beta^{\alpha}(\log (x))^{\alpha-1}}{x^{\beta+1} \Gamma(\alpha)}$, & $x \geq 1$ \\
Pareto & $\alpha>0, \nu>0$ & $f_{X}(x)=\frac{\alpha}{\nu+x}\left(\frac{\nu}{\nu+x}\right)^{\alpha}$, & $x \geq 0$ \\
Burr & $\alpha>0, \nu>0, \tau>0$ & $f_{X}(x)=\frac{\alpha \tau \nu^{\alpha} x^{\tau-1}}{\left(\nu+x^{\tau}\right)^{\alpha+1}}$, & $x \geq 0$ \\
\hline
\end{tabular}

The adjustment coefficient (also called the Lundberg exponent) plays a key role in calculating the ruin probability in the case of light-tailed claims. Suppose $\gamma=\sup _{z} M_{X}(z)<\infty$. A positive solution of the equation

$$
1+(1+\theta) \mu R=M_{X}(R), \quad R<\gamma,
$$

is called an adjustment coefficient.

An analytical solution to equation (3) exists only for few claim distributions. However, it is quite easy to obtain a numerical solution. The coefficient $R$ satisfies the inequality

$$
R<\frac{2 \theta \mu}{\mu^{(2)}}
$$

where $\mu^{(2)}=\mathbb{E} X_{i}^{2}$ (cf. Asmussen, 2000). Let $D(z)=1+(1+\theta) \mu z-M_{X}(z)$. Thus, the adjustment coefficient $R>0$ satisfies the equation $D(R)=0$. In order to get a solution one may use the Newton-Raphson formula 


$$
R_{j+1}=R_{j}-\frac{D\left(R_{j}\right)}{D^{\prime}\left(R_{j}\right)},
$$

with the initial condition $R_{0}=2 \theta \mu / \mu^{(2)}$, where $D^{\prime}(z)=(1+\theta) \mu-M_{X}(z)^{\prime}$.

Moreover, if it is possible to calculate the third raw moment $\mu^{(3)}$, we can obtain a sharper bound than (4) (see Panjer and Willmot, 1992):

$$
R<\frac{12 \mu \theta}{3 \mu^{(2)}+\sqrt{9\left(\mu^{(2)}\right)^{2}+24 \mu \mu^{(3)} \theta}},
$$

and use it as the initial condition in (5).

We note that most of the methods of estimating the ruin probability discussed in the paper require only the existence of first two or three moments of the claim size distribution, and some of them also the existence of the moment generating function.

Now, consider the aggregate loss process $S_{t}$ with $c=1$. Put $\xi(u)=S_{\tau(u)}-$ $u$, where $\tau(u)$ is the time to ruin defined by (1). The following statement presents a general formula for the ruin probability in infinite time (see e.g. Asmussen, 2000). Assume that for some $R>0$ the process $\left\{e^{R S_{t}}\right\}_{t \geq 0}$ is a martingale and $S_{t} \rightarrow-\infty$ a.s. on $\{\tau(u)=\infty\}$. Then

$$
\psi(u)=\frac{e^{-R u}}{\mathbb{E}\left(e^{R \xi(u)} \mid \tau(u)<\infty\right)} .
$$

For the classical risk model the foregoing assumptions hold and $R$ is an adjustment coefficient.

2. Exact ruin probabilities. Now, we are going to present a collection of basic exact results on the ruin probability in infinite time.

No initial capital. When $u=0$ it is easy to obtain the exact formula

$$
\psi(u)=\frac{1}{1+\theta} .
$$

For more details see e.g. Grandell (1991). Notice that the formula depends only on $\theta$, regardless of the claim size distribution.

Exponential claims. The explicit, easy-to-calculate formula exists for exponential claims, namely

$$
\psi(u)=\frac{1}{1+\theta} e^{\frac{-\theta \beta u}{1+\theta}} .
$$

Gamma claims. It was shown by Grandell and Segerdahl (1971) that for the gamma claim distribution with mean 1 and $\alpha \leq 1$,

$$
\psi(u)=\frac{\theta(1-R / \alpha) e^{-R u}}{1+(1+\theta) R-(1+\theta)(1-R / \alpha)}+\frac{\alpha \theta \sin (\alpha \pi)}{\pi} \cdot I,
$$


where

$$
I=\int_{0}^{\infty} \frac{x^{\alpha} e^{-(x+1) \alpha u} d x}{\left[x^{\alpha}(1+\alpha(1+\theta)(x+1))-\cos (\alpha \pi)\right]^{2}+\sin ^{2}(\alpha \pi)} .
$$

The integral $I$ has to be calculated numerically, but with some care near 0 this can be done precisely. We notice that the assumption on the mean is no restriction since for claims $X$ with arbitrary mean $\mu$ we have $\psi_{X}(u)=$ $\psi_{X / \mu}(u / \mu)$. As the gamma distribution is closed under scale changes we obtain $\psi_{G(\alpha, \beta)}(u)=\psi_{G(\alpha, \alpha)}(\beta u / \alpha)$ and we can now calculate the exact ruin probability via equation (8).

Mixture of $n$ exponentials. For the claim size distribution being a mixture of $n$ exponentials with parameters $\beta_{1}<\cdots<\beta_{n}$ and weights $a_{1}, \ldots, a_{n}$, using the Laplace transform inversion, one may obtain an exact formula of the form (Dufresne and Gerber, 1989)

$$
\psi(u)=\sum_{k=1}^{n} C_{k} e^{-r_{k} u},
$$

where $r_{1}, \ldots, r_{n}$ are $n$ positive solutions to the equation

$$
(1+\theta) \mu=\sum_{j=1}^{n} \frac{a_{j}}{\beta_{j}-r},
$$

with $0<r_{1}=R<\beta_{1}<r_{2}<\beta_{2}<\cdots<r_{n}<\beta_{n}$.

The coefficients $C_{k}$ are given by the formula

$$
C_{k}=\frac{1}{r_{k}} \frac{\sum_{j=1}^{n} \frac{a_{j}}{\beta_{j}-r_{k}}-\mu}{\sum_{j=1}^{n} \frac{a_{j}}{\left(\beta_{j}-r_{k}\right)^{2}}}
$$

In the case of mixture of two exponentials $(n=2)$ a simple analytic result is given (Panjer and Willmot, 1992):

$$
\psi(u)=\frac{1}{(1+\theta)\left(r_{2}-r_{1}\right)}\left\{\left(\varrho-r_{1}\right) \exp \left(-r_{1} u\right)+\left(r_{2}-\varrho\right) \exp \left(-r_{2} u\right)\right\}
$$

where

$$
\begin{aligned}
& r_{1}=\frac{\varrho+\theta\left(\beta_{1}+\beta_{2}\right)-\left[\left\{\varrho+\theta\left(\beta_{1}+\beta_{2}\right)\right\}^{2}-4 \beta_{1} \beta_{2} \theta(1+\theta)\right]^{1 / 2}}{2(1+\theta)}, \\
& r_{2}=\frac{\varrho+\theta\left(\beta_{1}+\beta_{2}\right)+\left[\left\{\varrho+\theta\left(\beta_{1}+\beta_{2}\right)\right\}^{2}-4 \beta_{1} \beta_{2} \theta(1+\theta)\right]^{1 / 2}}{2(1+\theta)}
\end{aligned}
$$

and

$$
\varrho=\beta_{1}(1-p)+\beta_{2} p, \quad p=\frac{a_{1} \beta_{1}^{-1}}{a_{1} \beta_{1}^{-1}+a_{2} \beta_{2}^{-1}} .
$$


3. A survey of approximations. When the claim size distribution is exponential (or closely related), simple analytic results for the ruin probability in infinite time are possible (see Section 2). For more general claim amount distributions, e.g. heavy-tailed, the Laplace transform technique does not work and one may need some estimates. In this section we present 12 different well known and not so well known approximations. We introduce a promising modification to the De Vylder approximation called the 3-moment gamma De Vylder approximation. Numerical comparison of the approximations is given in Section 4. We also note that new approximations have recently been proposed in the literature (see e.g. Lima et al., 2002 and Usábel, 2001), but as they work for specific classes of distributions and are far from computational simplicity, we will not consider them.

3.1. Cramér-Lundberg approximation. The following approximation holds:

$$
\psi_{\mathrm{CL}}(u)=C e^{-R u}, \quad \text { where } \quad C=\frac{\theta \mu}{M_{X}^{\prime}(R)-\mu(1+\theta)} .
$$

For the proof we refer to Grandell (1991). The classical Cramér-Lundberg approximation yields quite accurate results; however, we must remember that in order to use it an adjustment coefficient has to exist, therefore merely the light-tailed distributions can be taken into consideration.

For exponential claims formula (11) yields the exact result.

3.2. Exponential approximation. This approximation was proposed and derived by De Vylder (1996):

$$
\psi_{\mathrm{E}}(u)=\exp \left(-1-\frac{2 \mu \theta u-\mu^{(2)}}{\sqrt{\left(\mu^{(2)}\right)^{2}+(4 / 3) \theta \mu \mu^{(3)}}}\right) .
$$

3.3. Lundberg approximation. The following formula, called the Lundberg approximation, comes from Grandell (2000):

$$
\psi_{\mathrm{L}}(u)=\left[1+\left(\theta u-\frac{\mu^{(2)}}{2 \mu}\right) \frac{4 \theta \mu^{2} \mu^{(3)}}{3\left(\mu^{(2)}\right)^{3}}\right] e^{-2 \mu \theta u / \mu^{(2)}} .
$$

3.4. Beekman-Bowers approximation. The Beekman-Bowers approximation uses the following representation of the ruin probability:

$$
\psi(u)=\mathbb{P}(M>u)=\mathbb{P}(M>0) \mathbb{P}(M>u \mid M>0) .
$$

The idea of the approximation is to replace the conditional probability $1-\mathbb{P}(M>u \mid M>0)$ with a gamma distribution function $G(u)$ by fitting the first two moments (see Grandell, 2000). This leads to

$$
\psi_{\mathrm{BB}}(u)=\frac{1}{1+\theta}(1-G(u)),
$$


where the parameters $\alpha, \beta$ of $G$ are given by

$$
\alpha=\frac{1+\left(4 \mu \mu^{(3)} / 3\left(\mu^{(2)}\right)^{2}-1\right) \theta}{1+\theta}, \quad \beta=\frac{2 \mu \theta}{\mu^{(2)}+\left(4 \mu \mu^{(3)} / 3 \mu^{(2)}-\mu^{(2)}\right) \theta} .
$$

The Beekman-Bowers approximation gives rather accurate results, in the exponential case it becomes the exact formula. It can be used for distributions with finite first three moments, i.e. for example for exponential, gamma, lognormal, truncated normal and Weibull distributions. For loggamma distribution we have to set $\beta>3$, for Pareto distribution $\alpha>3$, and for Burr distribution $\alpha \tau>3$.

3.5. Rényi approximation. The Rényi approximation is based on a classical result about $p$-thinning, Rényi's theorem (see Grandell, 2000). It may also be derived from (12) when we replace the gamma distribution function $G$ with an exponential one, matching only the first moment. It could be regarded as a simplified version of the Beekman-Bowers approximation and is given by

$$
\psi_{\mathrm{R}}(u)=\frac{1}{1+\theta} e^{-2 \mu \theta u / \mu^{(2)}(1+\theta)} .
$$

3.6. De Vylder approximation. The idea of this approximation is to replace the risk process with the one with $\theta=\bar{\theta}, \lambda=\bar{\lambda}$ and exponential claims with parameter $\bar{\beta}$, fitting the first three moments (De Vylder, 1978).

Let

$$
\bar{\beta}=\frac{3 \mu^{(2)}}{\mu^{(3)}}, \quad \bar{\lambda}=\frac{9 \lambda \mu^{(2)^{3}}}{2 \mu^{(3)^{2}}}, \quad \bar{\theta}=\frac{2 \mu \mu^{(3)}}{3 \mu^{(2)^{2}}} \theta .
$$

Then De Vylder's approximation is given by

$$
\psi_{\mathrm{DV}}(u)=\frac{1}{1+\bar{\theta}} e^{-\overline{\theta \beta} u /(1+\bar{\theta})} .
$$

Obviously, in the exponential case the method gives the exact result. For other claim distributions in order to apply the approximation, similarly to the Beekman-Bowers approximation, the first three moments have to exist.

3.7. 3-moment gamma De Vylder approximation. Here we introduce a new approximation based on De Vylder's idea to replace the risk process with another one where the exact ruin probability is known. This time we switch to gamma distributed claims by fitting the first three moments and use the exact formula (8) for the ruin probability in the gamma case. However, the risk process with gamma claims is determined by four parameters $\left(\bar{\lambda}, \bar{\theta}, \bar{\mu}, \bar{\mu}^{(2)}\right)$, so now one additional equation is needed. Instead of the fourth raw moment, we propose $\bar{\mu}=\mu$. Since

$$
\begin{aligned}
& \mathbb{E} S_{t}=-\theta \lambda \mu t, \\
& \mathbb{E} S_{t}^{2}=\lambda \mu^{(2)} t+(\theta \lambda \mu t)^{2}, \\
& \mathbb{E} S_{t}^{3}=\lambda \mu^{(3)} t-3\left(\lambda \mu^{(2)} t\right)(\theta \lambda \mu t)-(\theta \lambda \mu t)^{2},
\end{aligned}
$$


and in the gamma case $\bar{\mu}^{(3)}=\frac{\bar{\mu}^{(2)}}{\bar{\mu}}\left(2 \bar{\mu}^{(2)}-\bar{\mu}^{2}\right)$, the parameters $\left(\bar{\lambda}, \bar{\theta}, \bar{\mu}, \bar{\mu}^{(2)}\right)$ must satisfy

$$
\mu=\bar{\mu}, \quad \theta \lambda \mu=\overline{\theta \lambda} \bar{\mu}, \quad \lambda \mu^{(2)}=\bar{\lambda} \bar{\mu}^{(2)}, \quad \lambda \mu^{(3)}=\bar{\lambda} \frac{\bar{\mu}^{(2)}}{\bar{\mu}}\left(2 \mu^{(2)}-\mu^{2}\right)
$$

and we get the solution

$$
\begin{gathered}
\bar{\lambda}=\frac{2 \lambda\left(\mu^{(2)}\right)^{2}}{\mu\left(\mu^{(3)}+\mu^{(2)} \mu\right)}, \quad \bar{\theta}=\frac{\theta \mu\left(\mu^{(3)}+\mu^{(2)} \mu\right)}{2\left(\mu^{(2)}\right)^{2}}, \\
\bar{\mu}=\mu, \quad \bar{\mu}^{(2)}=\frac{\mu\left(\mu^{(3)}+\mu^{(2)} \mu\right)}{2 \mu^{(2)}} .
\end{gathered}
$$

Finally, we obtain the approximation

$$
\psi_{3 \mathrm{MGDV}}(u)=\frac{\bar{\theta}(1-R / \bar{\alpha}) e^{-\bar{\beta} R u / \bar{\alpha}}}{1+(1+\bar{\theta}) R-(1+\bar{\theta})(1-R / \bar{\alpha})}+\frac{\bar{\alpha} \bar{\theta} \sin (\bar{\alpha} \pi)}{\pi} \cdot I,
$$

where

$$
I=\int_{0}^{\infty} \frac{x^{\bar{\alpha}} e^{-(x+1) \bar{\beta} u} d x}{\left[x^{\bar{\alpha}}(1+\bar{\alpha}(1+\bar{\theta})(x+1))-\cos (\bar{\alpha} \pi)\right]^{2}+\sin ^{2}(\bar{\alpha} \pi)},
$$

and $\bar{\alpha}=\bar{\mu}^{2} /\left(\bar{\mu}^{(2)}-\bar{\mu}^{2}\right), \bar{\beta}=\bar{\mu} /\left(\bar{\mu}^{(2)}-\bar{\mu}^{2}\right)$.

In the exponential and gamma case this method gives the exact result. For other claim distributions in order to apply the approximation, similarly to the De Vylder approximation, the first three moments have to exist. We will show that the method is a slight improvement on the De Vylder approximation which was said by Grandell (2000) to be the best among "simple" approximations.

3.8. Heavy traffic approximation. The term "heavy traffic" comes from queuing theory. In risk theory it means that on the average the premiums exceed only slightly the expected claims. This implies that the safety loading $\theta$ is positive and small. Asmussen (2000) suggests the following approximation:

$$
\psi_{\mathrm{HT}}(u)=\exp \left(-\frac{2 \theta \mu u}{\mu^{(2)}}\right) .
$$

This method requires the existence of the first two moments of the claim size distribution, so we assume: $\beta>2$ for the loggamma case, $\alpha>2$ for the Pareto case, and $\alpha \tau>2$ for the Burr case.

3.9. Light traffic approximation. As for heavy traffic, the term "light traffic" comes from queuing theory, but has an obvious interpretation also in risk theory, namely, on the average, the premiums are much larger than the expected claims. This implies that the safety loading $\theta$ is positive and large. 
We may obtain the following asymptotic formula:

$$
\psi_{\mathrm{LT}}(u)=\frac{1}{(1+\theta) \mu} \int_{u}^{\infty} \bar{F}_{X}(x) d x .
$$

In risk theory heavy traffic is most often argued to be the typical case rather than light traffic. However, light traffic is of some interest as a complement to heavy traffic; it is also needed for the interpolation approximation to be studied in the next subsection.

3.10. Heavy-light traffic approximation. The idea is to combine heavy and light approximations:

$$
\psi_{\mathrm{HLT}}(u)=\frac{\theta}{1+\theta} \psi_{\mathrm{LT}}\left(\frac{\theta u}{1+\theta}\right)+\frac{1}{(1+\theta)^{2}} \psi_{\mathrm{HT}}(u)
$$

(see Asmussen, 2000). The particular feature of this approximation is that it is exact for the exponential distribution and asymptotically correct both in light and heavy traffic.

3.11. Heavy-tailed claims approximation. First, let us introduce the class $\mathcal{S}$ of subexponential distributions (see e.g. Embrechts et al., 1997):

$$
\mathcal{S}=\left\{F: \lim _{x \rightarrow \infty} \frac{\overline{F^{* 2}}(x)}{\bar{F}(x)}=2\right\} \equiv\left\{F: \lim _{x \rightarrow \infty} \frac{\overline{F^{* n}}(x)}{\bar{F}(x)}=n, n \geq 2\right\} .
$$

This class contains lognormal and Weibull distributions (for $\tau<1$ ). Moreover, all distributions with a regularly varying tail (e.g. loggamma, Pareto and Burr distributions) are subexponential. For subexponential distributions we can formulate the following approximation of the ruin probability. If $F \in \mathcal{S}$, then the asymptotic formula for large $u$ is given by

$$
\psi_{\mathrm{HTC}}(u)=\frac{1}{\theta \mu}\left(\mu-\int_{0}^{u} \bar{F}(x) d x\right)
$$

(see Asmussen, 2000). This method can be used for Weibull, lognormal, loggamma, Pareto and Burr distributions.

3.12. Computer approximation via the Pollaczek-Khinchin formula. This time we use the representation (2) of the ruin probability and the decomposition of the maximum $M$ as a sum of ladder heights. Let $L_{1}$ be the value that the process $\left\{S_{t}\right\}$ reaches for the first time above the zero level. Next, let $L_{2}$ be the value which is obtained for the first time above the level $L_{1}$; $L_{3}, L_{4}, \ldots$ are defined in the same way. The values $L_{k}$ are called the ladder heights. Since the process $\left\{S_{t}\right\}$ has stationary and independent increments, $\left\{L_{k}\right\}_{k=1}^{\infty}$ is a sequence of independent and identically distributed variables. One can show that the number $K$ of ladder heights until ruin time is given by a geometric distribution with parameters $p=1 /(1+\theta)$ and $q=\theta /(1+\theta)$. 
Thus, the random variable $M$ may be expressed

$$
M=\sum_{i=1}^{K} L_{i}
$$

This implies that $M$ has a compound geometric distribution given by the distribution function

$$
F_{M}(x)=\frac{\theta}{1+\theta} \sum_{n=0}^{\infty} G^{* n}(x),
$$

where $G^{* n}$ is the $n$th convolution of the distribution with the defective density

$$
g(x)=\frac{1}{\mu(1+\theta)} \bar{F}_{X}(x)=\frac{1}{1+\theta} b_{0}(x),
$$

and the density

$$
b_{0}(x)=\frac{\bar{F}_{X}(x)}{\mu} .
$$

The above fact together with the representation (2) leads to the Pollaczek-Khinchin formula for the ruin probability:

$$
\psi(u)=\mathbb{P}(M>u)=\frac{\theta}{1+\theta} \sum_{n=0}^{\infty}\left(\frac{1}{1+\theta}\right)^{n} \overline{B_{0}^{* n}}(u),
$$

where $\bar{B}_{0}$ is the tail of the distribution function corresponding to the density $b_{0}$ and $\overline{B_{0}^{* 0}}(u) \equiv I_{\{u \geq 0\}}$.

One can use this formula to derive explicit solutions for a number of claim amount distributions (see e.g. Asmussen, 2000 or Panjer and Willmot, 1992). If that is not possible, the formula can be directly applied to calculating the ruin probability. However, it involves an infinite sum, hence we use the Monte Carlo method to simulate the random variable $M$ and calculate the ruin probability. From (15) the ruin probability $\psi(u)=E Z$, where $Z=1(M>u)$, may be generated as follows.

(1) Generate a random variable $K$ from the geometric distribution with parameters $p=1 /(1+\theta)$ and $q=\theta /(1+\theta)$.

(2) Generate random variables $X_{1}, \ldots, X_{K}$ from the density $b_{0}(x)$.

(3) Calculate $M=X_{1}+\cdots+X_{K}$.

(4) If $M>u$, let $Z=1$, otherwise let $Z=0$.

The main problem seems to be simulating random variables with the density $b_{0}(x)$.

Proposition 3.1. The density $b_{0}(x)$ has a closed form only for four of the distributions considered, namely 
(i) for exponential claims, $b_{0}(x)$ is the density of the same exponential distribution,

(ii) for mixture of exponential claims, $b_{0}(x)$ is the density of the mixture of exponential distributions with weights

$$
\left(\frac{a_{1} / \beta_{1}}{\sum_{i=1}^{n}\left(a_{i} / \beta_{i}\right)}, \ldots, \frac{a_{n} / \beta_{n}}{\sum_{i=1}^{n}\left(a_{i} / \beta_{i}\right)}\right)
$$

(iii) for Pareto claims, $b_{0}(x)$ is the density of the Pareto distribution with parameters $\alpha-1$ and $\nu$,

(iv) for Burr claims, $b_{0}(x)$ is the density of the transformed beta distribution.

Proof. (i) For exponential claims

$$
\bar{F}_{X}(x)=e^{-\beta x}, \quad \mu=\frac{1}{\beta},
$$

thus

$$
b_{0}(x)=\beta \bar{F}_{X}(x)=\beta e^{-\beta x},
$$

which yields again the exponential distribution, with parameter $\beta$.

(ii) For mixture of exponential claims

$$
\bar{F}_{X}(x)=\sum_{i=1}^{n} a_{i} e^{-\beta_{i} x}, \quad \mu=\frac{a_{1}}{\beta_{1}}+\cdots+\frac{a_{n}}{\beta_{n}},
$$

hence

$$
\begin{aligned}
b_{0}(x) & =\frac{1}{a_{1} / \beta_{1}+\cdots+a_{n} / \beta_{n}} \bar{F}_{X}(x) \\
& =\frac{a_{1}}{a_{1} / \beta_{1}+\cdots+a_{n} / \beta_{n}} \bar{F}_{X_{1}}(x)+\cdots+\frac{a_{n}}{a_{1} / \beta_{1}+\cdots+a_{n} / \beta_{n}} \bar{F}_{X_{n}}(x) \\
& =\frac{a_{1} / \beta_{1}}{a_{1} / \beta_{1}+\cdots+a_{n} / \beta_{n}} f_{X_{1}}(x)+\cdots+\frac{a_{n} / \beta_{n}}{a_{1} / \beta_{1}+\cdots+a_{n} / \beta_{n}} f_{X_{n}}(x),
\end{aligned}
$$

which is again a mixture of exponential distributions, with weights

$$
\left(\frac{a_{1} / \beta_{1}}{\sum_{i=1}^{n}\left(a_{i} / \beta_{i}\right)}, \cdots, \frac{a_{n} / \beta_{n}}{\sum_{i=1}^{n}\left(a_{i} / \beta_{i}\right)}\right) \text {. }
$$

(iii) For Pareto claims

$$
\bar{F}_{X}(x)=\left(\frac{\nu}{\nu+x}\right)^{\alpha}, \quad \mu=\frac{\nu}{\alpha-1}, \quad \alpha>1,
$$

so

$$
b_{0}(x)=\frac{\alpha-1}{\nu} \bar{F}_{X}(x)=\frac{\alpha-1}{\nu}\left(\frac{\nu}{\nu+x}\right)^{\alpha}=\frac{\alpha-1}{\nu+x}\left(\frac{\nu}{\nu+x}\right)^{\alpha-1},
$$

which again gives the Pareto distribution with parameters $(\alpha-1, \nu)$. 
(iv) For Burr claims

$$
\bar{F}_{X}(x)=\left(\frac{\nu}{\nu+x^{\tau}}\right)^{\alpha}, \quad \mu=\nu^{1 / \tau} \frac{\Gamma(\alpha-1 / \tau) \Gamma(1+1 / \tau)}{\Gamma(\alpha)}, \quad \alpha \tau>1,
$$

therefore

$$
b_{0}(x)=\frac{\Gamma(\alpha)}{\nu^{1 / \tau} \Gamma(\alpha-1 / \tau) \Gamma(1+1 / \tau)}\left(\frac{\nu}{\nu+x^{\tau}}\right)^{\alpha}
$$

Set

$$
a=\alpha-1 / \tau, \quad b=1 / \tau, \quad c=\tau, \quad d=\nu
$$

Then

$$
\begin{aligned}
b_{0}(x) & =\frac{\Gamma(a+b)}{d^{b} \Gamma(a) \Gamma(1+b)}\left(\frac{d}{d+x^{c}}\right)^{a+b}=\frac{\Gamma(a+b) d^{a}}{\Gamma(a) b \Gamma(b)\left(d+x^{c}\right)^{a+b}} \\
& =\frac{\Gamma(a+b) c d^{a} x^{c b-1}}{\Gamma(a) \Gamma(b)\left(d+x^{c}\right)^{a+b}} .
\end{aligned}
$$

The foregoing formula represents the density of the transformed beta distribution with parameters $a, b, c$ and $d$. This distribution comes as a quotient of two variables with generalized gamma distribution with corresponding parameters (for details see Panjer and Willmot, 1992).

For other distributions treated in this paper, in order to generate random variables $X_{k}$ we use formula (14) and controlled, numerical integration. The above described computer approximation via the Pollaczek-Khinchin for-

Table 2. Survey of approximations with an indication when they can be applied

\begin{tabular}{ccccccccc}
\hline & \multicolumn{7}{c}{ Distribution } \\
\cline { 2 - 8 } Method & Exp. & Gam. & $\begin{array}{c}\text { Wei- } \\
\text { bull }\end{array}$ & $\begin{array}{c}\text { Mix. } \\
\text { Exp. }\end{array}$ & $\begin{array}{c}\text { Log- } \\
\text { norm. }\end{array}$ & $\begin{array}{c}\text { Log- } \\
\text { gam. }\end{array}$ & Pareto & Burr \\
\hline Cramér-Lundberg & + & + & - & + & - & - & - & - \\
Exponential & + & + & + & + & + & $\beta>3$ & $\alpha>3$ & $\alpha \tau>3$ \\
Lundberg & + & + & + & + & + & $\beta>3$ & $\alpha>3$ & $\alpha \tau>3$ \\
Beekman-Bowers & + & + & + & + & + & $\beta>3$ & $\alpha>3$ & $\alpha \tau>3$ \\
Rényi & + & + & + & + & + & $\beta>2$ & $\alpha>2$ & $\alpha \tau>2$ \\
De Vylder & + & + & + & + & + & $\beta>3$ & $\alpha>3$ & $\alpha \tau>3$ \\
3M Gam. De Vylder & + & + & + & + & + & $\beta>3$ & $\alpha>3$ & $\alpha \tau>3$ \\
Heavy traffic & + & + & + & + & + & $\beta>2$ & $\alpha>2$ & $\alpha \tau>2$ \\
Light traffic & + & + & + & + & + & + & + & + \\
Heavy-light traffic & + & + & + & + & + & $\beta>2$ & $\alpha>2$ & $\alpha \tau>2$ \\
Heavy-tailed & - & - & $\tau<1$ & - & - & + & + & + \\
Pollaczek-Khinchin & + & + & + & + & + & + & + & + \\
\hline
\end{tabular}


mula will be briefly called the Pollaczek-Khinchin approximation. We note that the approximation works for all distributions of claims considered.

3.13. Summary of the approximations. Table 2 shows which approximation can be used for a particular choice of claim size distribution. Moreover, the necessary assumptions on the distribution parameters are included.

4. Numerical comparison of the methods. We now aim to compare the 12 approximations presented in the preceding section. To this end we consider the ruin probability as a function of the initial capital $u$, with different claim amount distributions. In order to show the relative errors of the methods we compare results of the approximations with the exact values, which can be done in the exponential, gamma and mixture-of-exponentials case, partially in the lognormal case, or with the results obtained via the Pollaczek-Khinchin formula, which we feel, and justify it numerically, can be a reference method. In the Pollaczek-Khinchin approximation, for the Monte Carlo method purposes, we generate 100 blocks of 100000 simulations. The simulated ruin probabilities result in $95 \%$ confidence intervals with length at most $6 \cdot 10^{-4}$.

For the exponential case the Cramér-Lundberg, Rényi, Beekman-Bowers, De Vylder and 3-moment gamma De Vylder approximations yield the exact result given by formula (7). We study other approximations for $\theta=0.01$ (very small value), $\theta=5$ (very large value) and $\theta=1$ (large value).

In the figures below, diagram (a) represents the ruin probability, and (b) the relative error of the approximations (with respect to the exact value, the exponential approximation or the Pollaczek-Khinchin approximation).

First, we consider the case when $\theta=0.01$. As we can clearly see in Figure 1(a), the light traffic approximation shows a total lack of accuracy. Figure 1(b) suggests that exponential and Lundberg approximations work

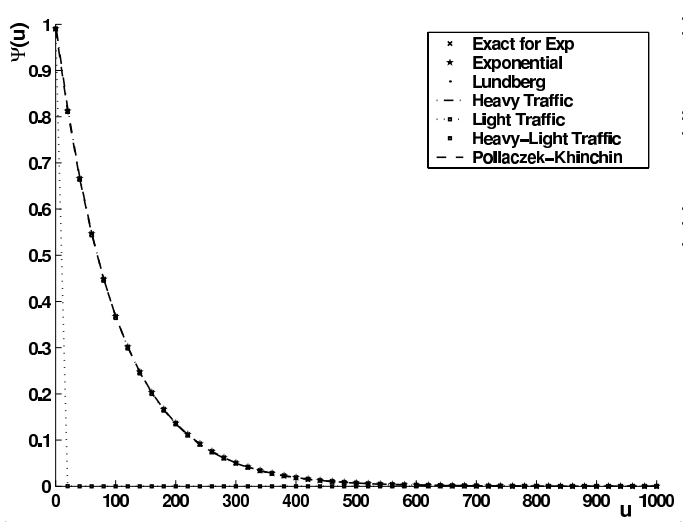

(a)

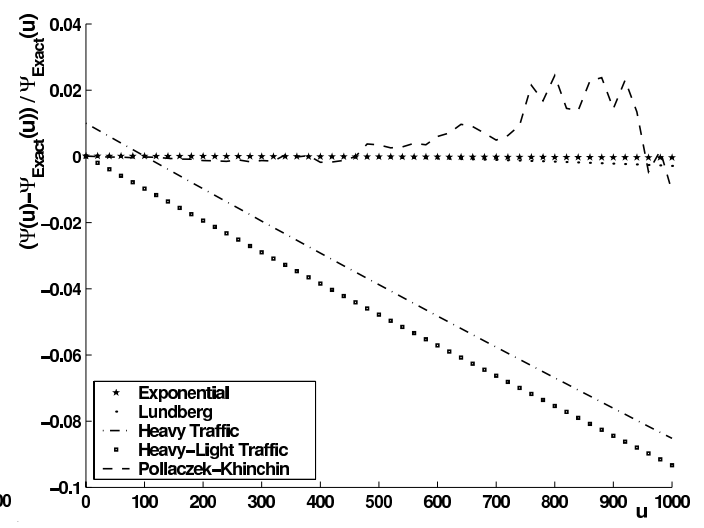

(b)

Fig. 1. The exponential case with $\beta=1, \theta=0.01$ and $u \leq 1000$ 


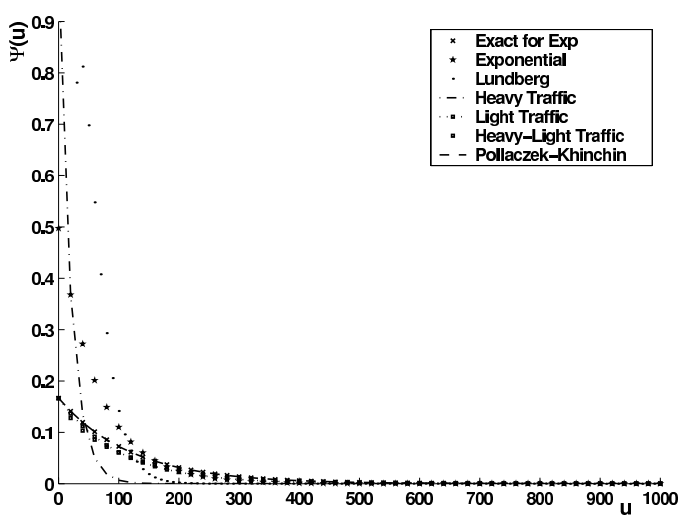

(a)

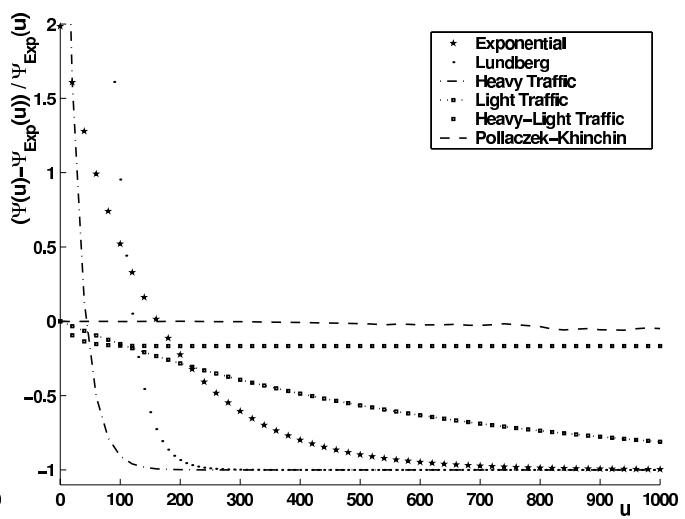

(b)

Fig. 2. The exponential case with $\beta=0.01, \theta=5$ and $u \leq 1000$

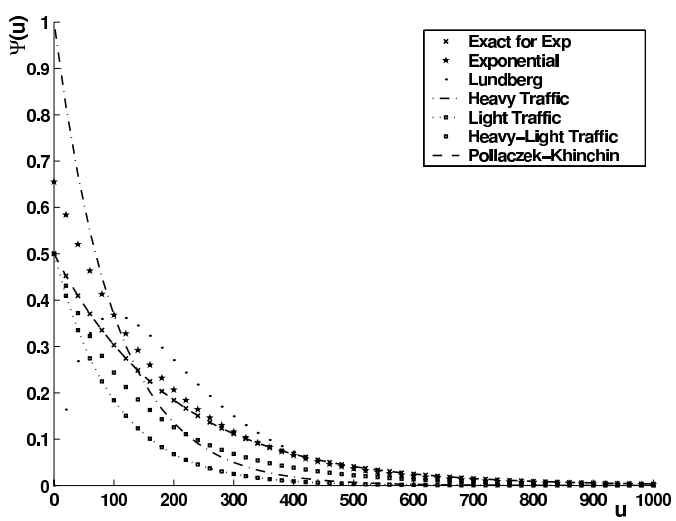

(a)

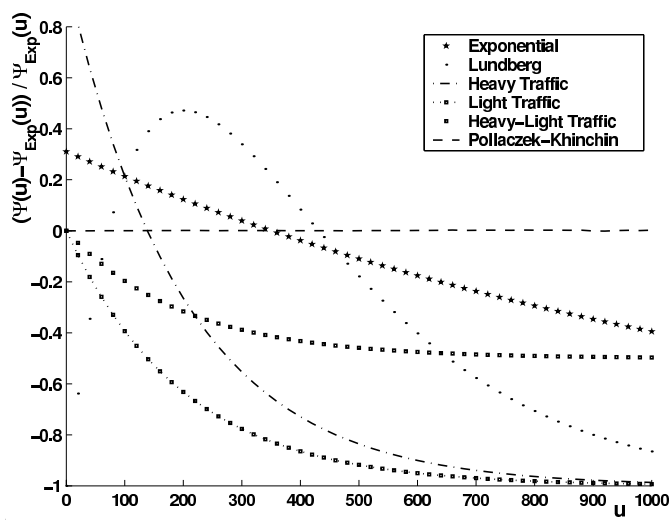

(b)

Fig. 3. The exponential case with $\beta=0.01, \theta=1$ and $u \leq 1000$

extremely well, the Pollaczek-Khinchin formula holds a good accuracy and the rest of the methods do not work so well. However, looking at the $y$-axis scale (limits from $-10 \%$ to $4 \%$ ) we may say that all methods except the light traffic give accurate results.

When $\theta=5$ (see Figure 2), the Pollaczek-Khinchin formula works very well. The heavy-light traffic approximation comes second. The other approximations are clearly unacceptable.

In the case $\theta=1$ (see Figure 3), the Pollaczek-Khinchin formula works extremely well, the other approximations hold little accuracy.

Hereafter in this section we will usually assume that the mean of the claim distribution is equal to 1 and $\theta=0.1$.

In the gamma case we can obtain exact values via formula (8) and use them in order to compare all methods except the heavy-tailed and 


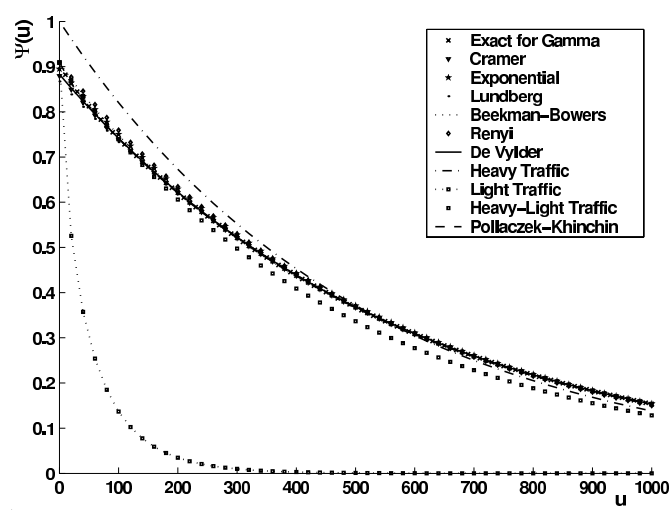

(a)

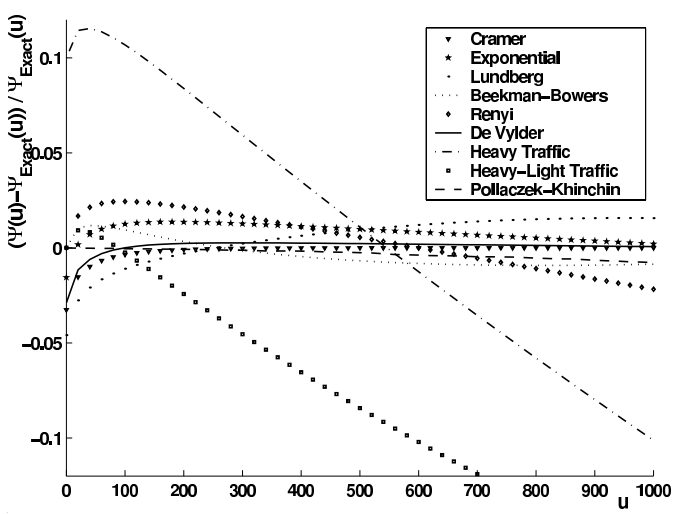

(b)

Fig. 4. The gamma case with $\alpha=0.01, \beta=0.01, \theta=0.1$ and $u \leq 1000$

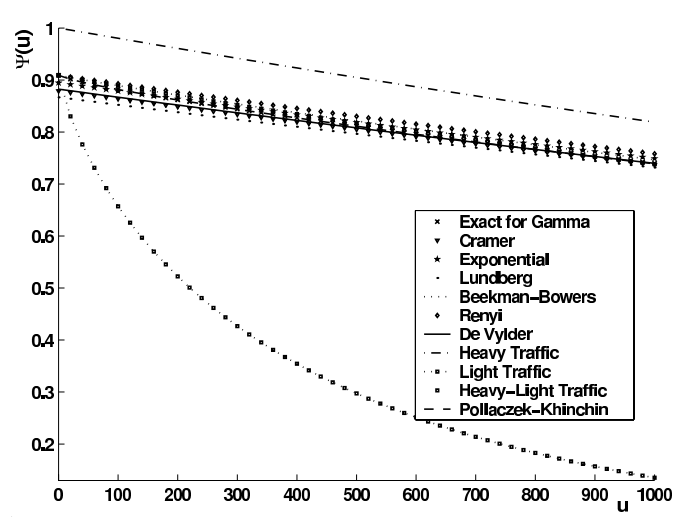

(a)

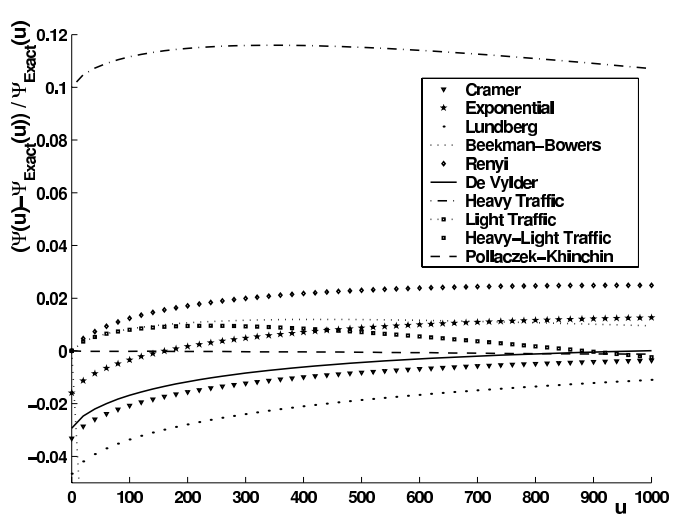

(b)

Fig. 5. The gamma case with $\alpha=0.001, \beta=0.001, \theta=0.1$ and $u \leq 1000$

3-moment gamma De Vylder approximations, which yield the exact result. When $\alpha=0.01$ and $\beta=0.01$ (see Figure 4), all approximations except the heavy, light (disastrous results) and heavy-light traffic, give a relative error of less than $3 \%$. When we increase the variance $(\alpha=0.001$ and $\beta=0.001$, see Figure 5), the situation is similar, but this time the heavy-light traffic approximation looks much better and the Pollaczek-Khinchin formula comes first with almost exact results.

When the claim distribution is a mixture of three exponentials (see Figure 6), the Cramér-Lundberg, De Vylder, 3-moment gamma De Vylder and exponential approximations give quite accurate results, while the BeekmanBowers and Lundberg approximations are just acceptable.

Since there are no exact methods for other distributions considered, we are going to calculate the relative errors with respect to the most accurate method. From Figures 1-6 the possible candidates are the Cramér- 


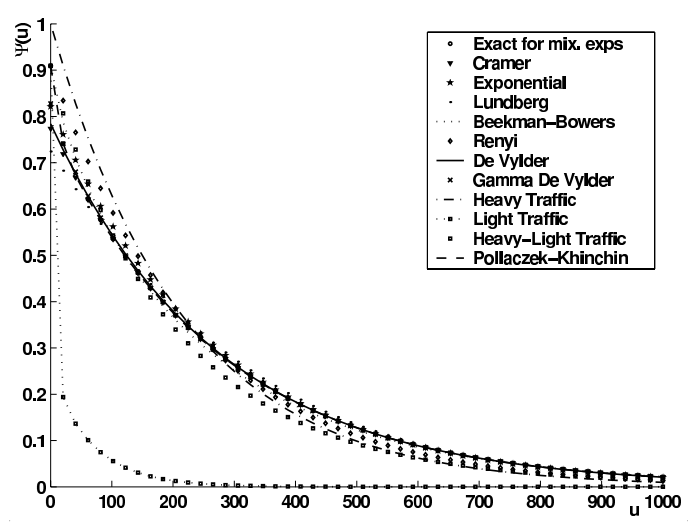

(a)

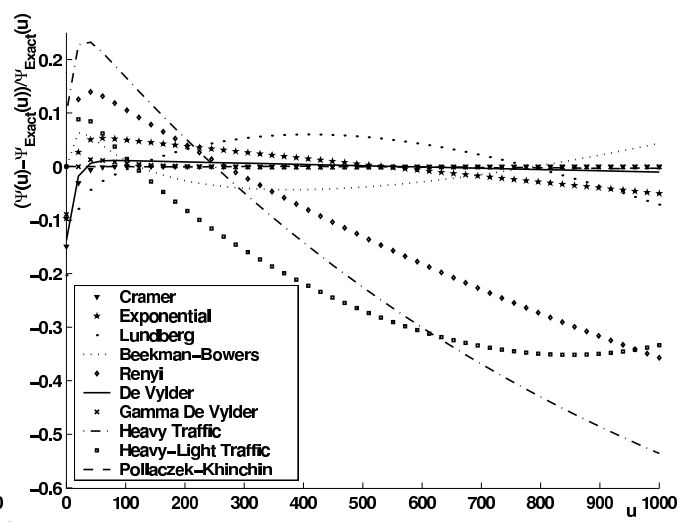

(b)

Fig. 6. The mixture of three exponentials with $\beta_{1}=0.014631, \beta_{2}=0.190206, \beta_{3}=$ 5.514588 , weight $a_{1}=0.0039793, a_{2}=0.1078392, a_{3}=0.8881815, \theta=0.1$ and $u \leq 1000$

Table 3. Comparison of the De Vylder, 3-moment gamma De Vylder and Pollaczek-Khinchin approximations under the mixture of three exponentials. Relative errors in $\%$.

\begin{tabular}{rrrrrr}
\hline$\theta$ & \multicolumn{1}{c}{$u$} & $\psi(u)$ & \multicolumn{1}{c}{$E_{\mathrm{PK}}$} & \multicolumn{1}{c}{$E_{\mathrm{DV}}$} & $E_{3 \mathrm{MGDV}}$ \\
\hline 0.05 & 10 & 0.8897 & 0.0151 & -3.2089 & -1.6062 \\
0.10 & 10 & 0.7993 & -0.0281 & -5.4247 & -2.6773 \\
0.15 & 10 & 0.7242 & -0.0320 & -6.9981 & -3.4102 \\
0.20 & 10 & 0.6611 & 0.0075 & -8.1485 & -3.8859 \\
0.25 & 10 & 0.6073 & $-\mathbf{0 . 0 0 2 8 0}$ & $-\mathbf{8 . 9 7 9 1}$ & $-\mathbf{4 . 1 8 7 4}$ \\
0.30 & 10 & 0.5610 & -0.0366 & -9.5811 & -4.3583 \\
1.00 & 10 & 0.2634 & -0.0566 & -10.6644 & -2.9803 \\
\hline 0.05 & 100 & 0.7144 & $-\mathbf{0 , 0 0 1 8}$ & $\mathbf{0 . 3 7 3 7}$ & $\mathbf{0 . 2 9 6 7}$ \\
0.10 & 100 & 0.5393 & -0.0170 & 1.1125 & 0.6082 \\
0.15 & 100 & 0.4247 & -0.0590 & 1.9143 & 0.8429 \\
0.20 & 100 & 0.3455 & 0.0179 & 2.7120 & 1.0304 \\
0.25 & 100 & 0.2886 & 0.0237 & 3.3784 & 1.1019 \\
0.30 & 100 & 0.2461 & 0.0264 & 3.9862 & 1.1418 \\
1.00 & 100 & 0.0724 & -0.2033 & 7.2086 & -0.0773 \\
\hline 0.05 & 1000 & 0.1149 & -0.0159 & 0.0087 & -0.0087 \\
0.10 & 1000 & 0.0210 & -0.2071 & -0.9429 & 0.0190 \\
0.15 & 1000 & 0.0054 & -0.7574 & -3.7000 & -0.2889 \\
0.20 & 1000 & 0.0018 & -3.5056 & -8.4000 & -1.3167 \\
0.25 & 1000 & 0.0007 & $\mathbf{0 . 2 7 1 4}$ & $-\mathbf{1 0 . 5 7 1 4}$ & $\mathbf{1 . 4 3 4 3}$ \\
0.30 & 1000 & 0.0003 & 7.7333 & -8.8700 & 9.7900 \\
1.00 & 1000 & 0.0000 & & & - \\
\hline & & & & & -
\end{tabular}


Table 4. Comparison of De Vylder, 3-moment gamma De Vylder and Pollaczek-Khinchin approximations under the lognormal distribution. Relative errors in \%.

\begin{tabular}{rrrrrr}
\hline$\theta$ & $u$ & $\psi(u)$ & \multicolumn{1}{c}{$E_{\mathrm{PK}}$} & \multicolumn{1}{c}{$E_{\mathrm{DV}}$} & \multicolumn{1}{c}{$E_{3 \mathrm{MGDV}}$} \\
\hline 0.05 & 100 & 0.55074 & -0.0182 & -20.6159 & -19.2468 \\
0.10 & 100 & 0.34395 & $\mathbf{0 . 0 0 8 7}$ & $\mathbf{- 1 9 . 4 8 2 5}$ & $\mathbf{- 1 8 . 4 8 5 2}$ \\
0.15 & 100 & 0.23573 & -0.02545 & -14.2281 & -13.6088 \\
0.20 & 100 & 0.17309 & 0.0983 & -8.0883 & -7.8052 \\
0.25 & 100 & 0.13384 & 0.0448 & -2.0547 & -2.0622 \\
0.30 & 100 & 0.10765 & 0.21367 & 3.5300 & 3.2606 \\
1.00 & 100 & 0.02535 & 0.0789 & 41.7278 & 39.6726 \\
\hline 0.05 & 1000 & 0.04199 & 2.6530 & 55.0917 & 53.1769 \\
0.10 & 1000 & 0.01099 & 4.0218 & 85.5323 & 87.9436 \\
0.15 & 1000 & 0.00574 & 3.92334 & 79.6690 & 87.7003 \\
0.20 & 1000 & 0.00384 & $\mathbf{2 . 7 9 1 7}$ & $\mathbf{6 8 . 6 9 7 9}$ & $\mathbf{8 1 . 0 3 6 5}$ \\
0.25 & 1000 & 0.00288 & 3.2431 & 59.1632 & 74.5937 \\
0.30 & 1000 & 0.00230 & 3.3304 & 51.8174 & 69.5435 \\
1.00 & 1000 & 0.00060 & 6.3000 & 19.8233 & 46.7283 \\
\hline 0.05 & 10000 & 0.00008 & $\mathbf{2 . 5 0 0 0}$ & $-\mathbf{9 9 . 9 9 9 6}$ & $-\mathbf{9 9 . 9 9 9}$ \\
0.10 & 10000 & 0.00004 & -9.5000 & -100.0000 & -100.0000 \\
0.15 & 10000 & 0.00002 & 27.0000 & -100.0000 & -100.0000 \\
0.20 & 10000 & 0.00002 & -15.0000 & -100.0000 & -100.0000 \\
0.25 & 10000 & 0.00001 & 50.0000 & -100.0000 & -100.0000 \\
0.30 & 10000 & 0.00001 & 22.0000 & -100.0000 & -100.0000 \\
1.00 & 10000 & 0.00000 & - & & - \\
\hline & & & & &
\end{tabular}

Lundberg, De Vylder, 3-moment gamma De Vylder and Pollaczek-Khinchin approximations. However, the Cramér-Lundberg approximation works only for light-tailed distributions, hence we have to choose between the De Vylder and Pollaczek-Khinchin approximations.

To this end we take into consideration a mixture of three exponential distributions and a lognormal distribution. For simple analytic results in the former case see Section 2. In the latter case, with a choice of specific parameters, exact values of the ruin probability can be computed using numerical inversion of the Laplace transform (see Wikstad, 1971, and Thorin and Wikstad, 1977). Let us now compare the three approximations. In Tables 3 and 4 the exact and approximate values, and relative errors are shown with respect to $u$ and $\theta$ with the distribution parameters as in Grandell and Segerdahl (1971); cf. Figures 6 and 8.

It is easy to notice that De Vylder and 3-moment gamma De Vylder are no match for the Pollaczek-Khinchin approximation (see the boldface 
results). This and Figures 1-6 justify the statement that the PollaczekKhinchin approximation can be chosen as a reference method. Moreover, it is worth noting that in the light-tailed cases the 3-moment gamma De Vylder approximation gives much more accurate results than the original method. Henceforth we will compare the methods with respect to the values obtained via the Pollaczek-Khinchin formula.

\section{Pollaczek-Khinchin approximation as the reference method.}

For the Weibull case (see Figure 7), the De Vylder, 3-moment gamma De Vylder, exponential and Lundberg approximations produce an error from about $-25 \%$ to $15 \%$. However, the most accurate results are obtained with the Beekman-Bowers approximation (error less than 4\%).

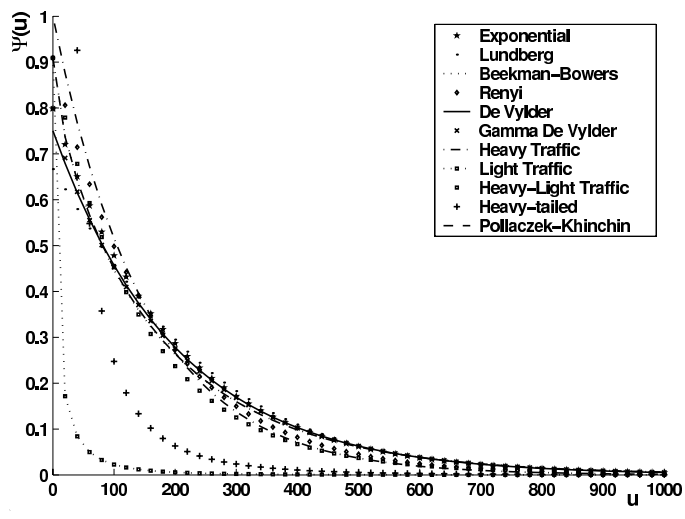

(a)

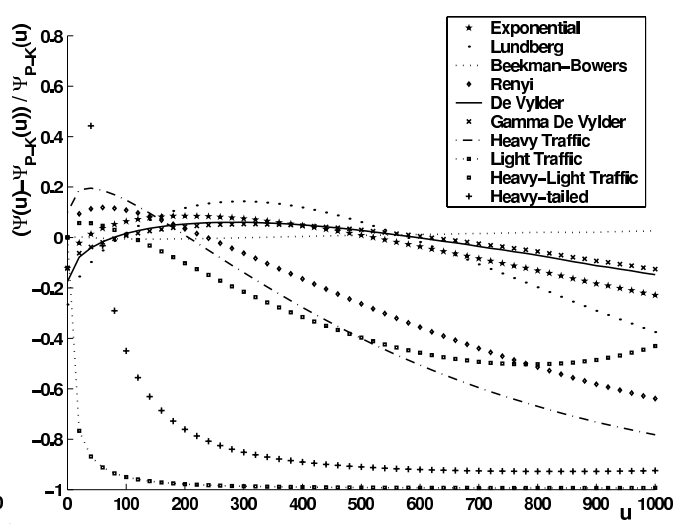

(b)

Fig. 7. The Weibull case with $c=1.9498, \tau=0.3, \theta=0.1$ and $u \leq 1000$

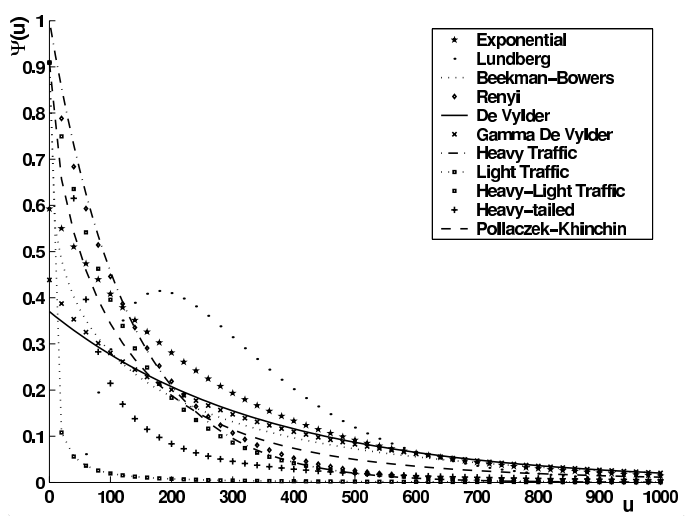

(a)

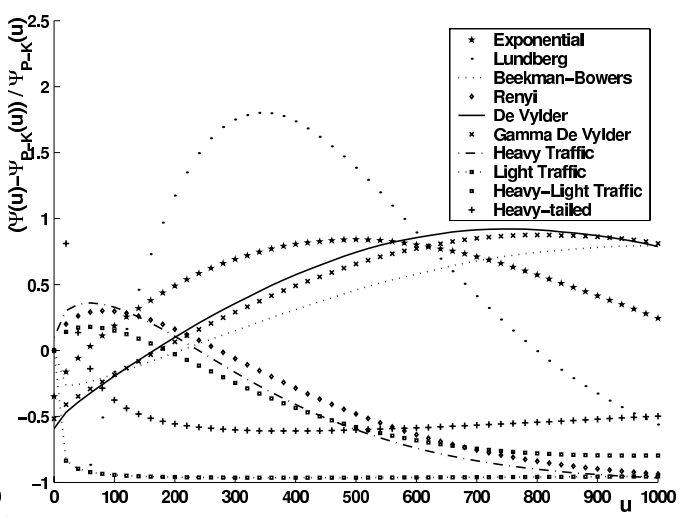

(b)

Fig. 8. The lognormal case with $\mu=-1.62, \sigma=1.8, \theta=0.1$ and $u \leq 1000$ 


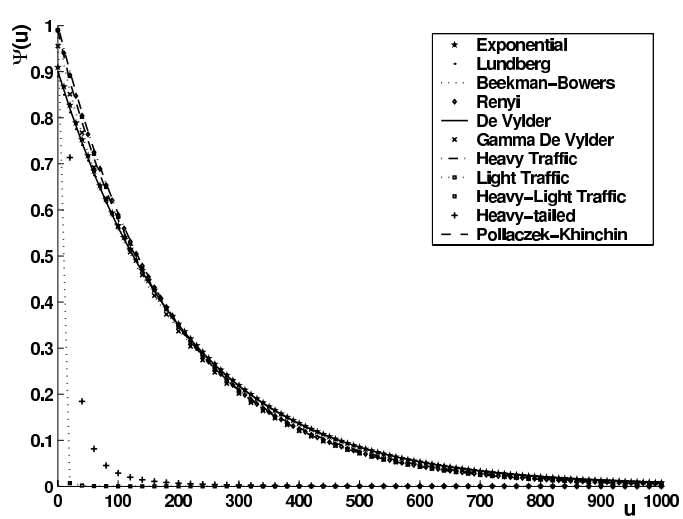

(a)

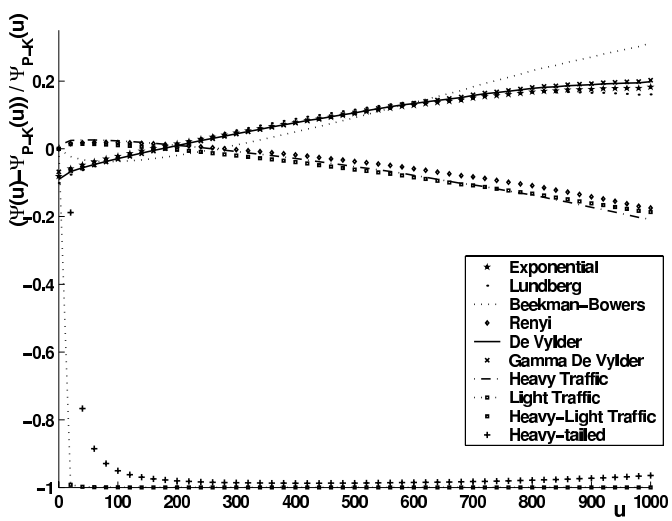

(b)

Fig. 9. The Pareto case with $\alpha=3.1, \nu=2.1, \theta=0.01$ and $u \leq 1000$

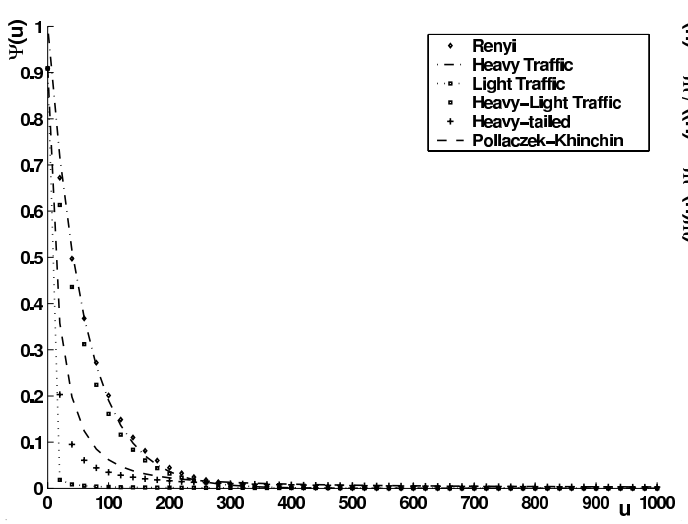

(a)

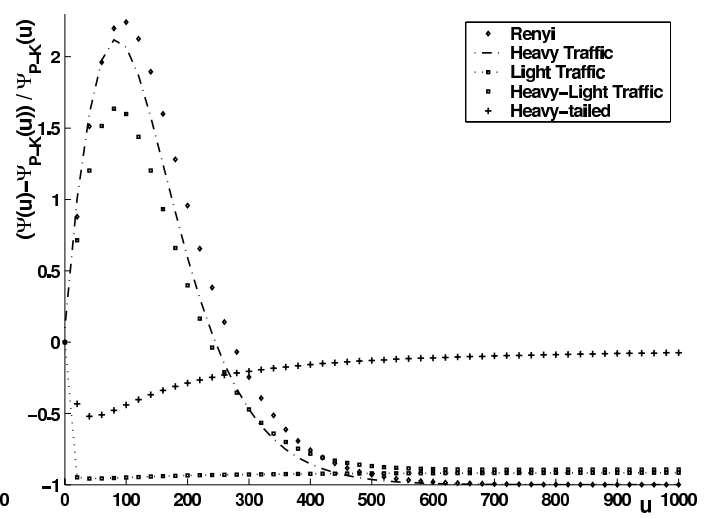

(b)

Fig. 10. The Burr case with $\alpha=1.4, \nu=0.6987, r=1.5, \theta=0.1$ and $u \leq 1000$

In the lognormal case (see Figure 8), the situation is very interesting. All methods give an error greater than $50 \%$. The lognormal case is quite important as loss data often appear to have the lognormal distribution. Thus we may say that using the Pollaczek-Khinchin approximation is essential when dealing with real-life data.

For the Pareto distributed claims (see Figure 9), all methods produce an error of up to about 20\%, light traffic and heavy-tailed approximations show a total lack of accuracy. The parameters of the Pareto distribution imply that the first three moments still exist. Now, we switch to the case when that is not true. 


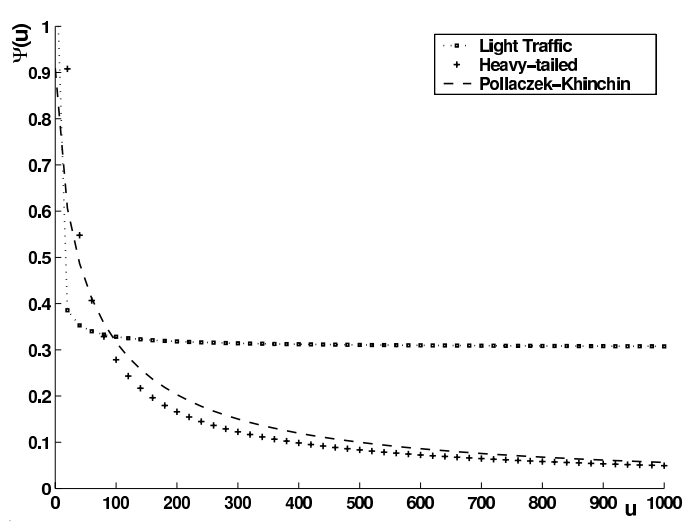

(a)

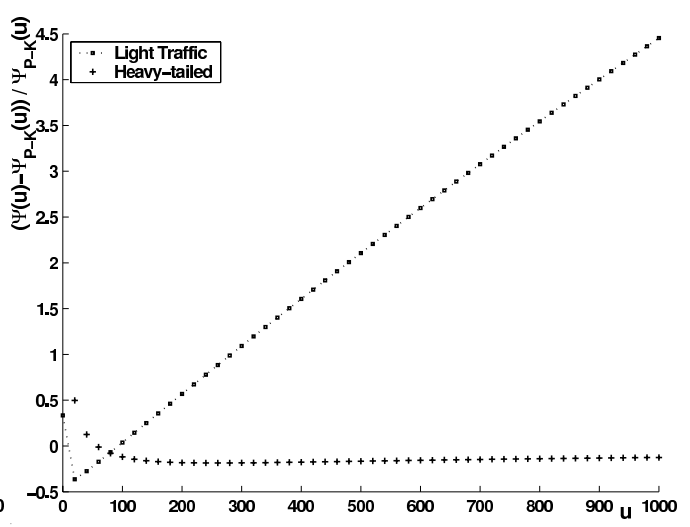

(b)

Fig. 11. The loggamma case with $\alpha=1.2, \beta=1.8, \theta=0.1$ and $u \leq 1000$

For the heavier case (see Figure 11), when the claim distribution is Burr and only the first two moments exist, we cannot use the exponential, Lundberg, Beekman-Bowers, De Vylder and 3-moment gamma De Vylder approximations. The rest show a total lack of accuracy, except for the heavy-tailed approximation which for greater values of $u$ seems to produce an error of up to $10 \%$.

In Figure 11 the very heavy-tailed case is considered, i.e. the loggamma distribution with only the first moment finite (the mean still exists). This time the parameters of the distribution cannot be matched in such a way that the mean is equal to 1 . The mean is now about 2.65. In that case merely light traffic and heavy-tailed approximations still work, and again the heavy-tailed approximation definitely comes first.

6. Final conclusions. The main finding of the paper is that the approximation via the Pollaczek-Khinchin formula is the best method for calculating the ruin probability in infinite time:

- Only two of the 12 approximations considered work for all distributions, namely Pollaczek-Khinchin and light traffic. From Figures 1-6 it is clear that the former is much better.

- Figures 1-6 demonstrate that among all the presented approximations which work for the light- and heavy-tailed distributions only De Vylder, 3-moment gamma De Vylder and Pollaczek-Khinchin behave well.

- In Tables 3 and 4 the exact and approximate values of the three approximations, and relative errors with respect to $u$ and $\theta$, are shown. It is easy to notice that both De Vylder approximations are no match for the Pollaczek-Khinchin approximation (see the boldface results). This 
and Figures 1-6 justify the statement that the Pollaczek-Khinchin approximation can be chosen as the reference method. Moreover, the 3 -moment gamma De Vylder approximation produces more accurate results than the original method.

- The Pollaczek-Khinchin approximation gives the most accurate results, even for the class of heavy-tailed distributions like lognormal. We also note that in each case for the Monte Carlo method purposes we generated 100 blocks of 100000 simulations and the variance within the results derived from the blocks was always relatively small.

Finally, let us remark that methods of approximation of the ruin probability in finite time are different from the ones in infinite time (see Asmussen, 2000, and Furrer et al., 1997). A detailed study will appear in a separate paper.

Acknowledgments. This research was supported by the State Committee for Scientific Research (KBN) Grant No. PBZ-KBN 016/P03/1999. The research of the third author was partly supported by KBN Grant No. 4T10B03025. The authors are grateful to two referees for their critical comments leading to the improvement of the paper.

\section{References}

S. Asmussen (2000), Ruin Probabilities, World Sci., Singapore.

F. E. De Vylder (1978), A practical solution to the problem of ultimate ruin probability, Scand. Actuar. J., 114-119.

F. E. De Vylder (1996), Advanced Risk Theory. A Self-Contained Introduction, Éditions de l'Université de Bruxelles and Swiss Association of Actuaries.

F. Dufresne and H. U. Gerber (1989), Three methods to calculate the probability of ruin, Astin Bull. 19, 71-90.

P. Embrechts, C. Klüppelberg and T. Mikosch (1997), Modelling Extremal Events for Insurance and Finance, Springer, Berlin.

H. Furrer, Z. Michna and A. Weron (1997), Stable Lévy motion approximation in collective risk theory, Insurance Math. Econom. 20, 97-114.

J. Grandell and C.-O. Segerdahl (1971), A comparison of some approximations of ruin probabilities, Skand. Aktuarietidskr., 144-158.

J. Grandell (1991), Aspects of Risk Theory, Springer, New York.

J. Grandell (2000), Simple approximations of ruin probability, Insurance Math. Econom. 26, 157-173.

F. Lima, J. Garcia and A. Egidio dos Reis (2002), Fourier/Laplace transforms and ruin probabilities, Astin Bull. 32, 91-105.

H. H. Panjer and G. E. Willmot (1992), Insurance Risk Models, Society of Actuaries, Schaumburg.

T. Rolski, H. Schmidli, V. Schmidt and J. Teugels (1999), Stochastic Processes for Insurance and Finance, Wiley, Chichester. 
O. Thorin and N. Wikstad (1977), Calculation of ruin probabilities when the claim distribution is lognormal, Astin Bull. 9, 231-246.

M. Usábel (2001), Ultimate ruin probabilities for generalized gamma-convolutions claim sizes,ibid. 31, 59-79.

N. Wikstad (1971), Exemplification of ruin probabilities, ibid. 6, 147-152.

Hugo Steinhaus Center

for Stochastic Methods

Institute of Mathematics

Wrocław University of Technology

Wyspiańskiego 27

50-370 Wrocław, Poland

E-mail: burnecki@im.pwr.wroc.pl

mista@im.pwr.wroc.pl

aleksander.weron@pwr.wroc.pl
Institute of Power Systems Automation

Wystawowa 1

51-618 Wrocław, Poland

Received on 7.7.2003;

revised version on 2.9.2004 